\title{
HIGH TEMPERATURE SYMMETRY NONRESTORATION
}

\author{
BORUT BAJC \\ Department of Physics, New York University, New York, NY 10003, USA \\ E-mail: bb46@is2.nyu.edu \\ and \\ J. Stefan Institute, Jamova 39, 1001 Ljubljana, Slovenia \\ E-mail: borut.bajc@ijs.si
}

\begin{abstract}
This is a short review on the subject of symmetry nonrestoration at high temperature. Special emphasis is put on experimental discoveries and different theoretical mechanisms. At the end, possible cosmological applications are briefly mentioned.
\end{abstract}

\section{Introduction}

Naively one expects that at low temperature a system has less symmetry than at high temperature. However, there are cases in nature, where the opposite happens. This phenomenon is called inverse symmetry breaking.

A similar phenomenon is symmetry nonrestoration at high temperature. It appears when the system has at high $T$ less symmetry than allowed by the Lagrangian (some vev is nonzero). With high temperature we mean temperature higher than any parameter of mass dimension in the Lagrangian.

In this short review we will describe some examples of this phenomenon both in nature and in field theory. Due to lack of space many topics will be mentioned only briefly. The interested reader can refer to some other reviews 1 as well as to the original papers.

\section{Experimental signatures}

We will describe here two examples in nature, which exhibit the strange phenomenona of symmetry nonrestoration orinverse symmetry breaking.

The Rochelle Salt. The system $\mathrm{Al}_{3}$ has in the interesting regime two critical temperatures. Below $T_{c 1}=-18^{\circ} \mathrm{C}$ and above $T_{c 2}=24^{\circ} \mathrm{C}$ the unit cell of the Rochelle salt is orthorhombic, while it is monoclinic in between. Since the orthorhombic unit cell is more symmetric than the monoclinic one, it is the phase transition at the lower critical temperature $T_{c 1}$ to be counterintuitive: heating the system we get a less symmetric object. Of course the next phase transition at $T_{c 2}$ restores again the symmetry, and symmetry nonrestoration is thus present only in the interval between $T_{c 1}$ and $T_{c 2}$.

Liquid Crystals $\left(\mathbf{S m C}^{*}\right)$. The second example of inverse symmetry breaking, or, as it is called in condensed matter, of re-entrant phase behavior, 
is relatively recent. The existence of the phase was argued in 1995 and later experimentally found in 1998.

First, why is the system called SmC*? Sm stays for smectic, i.e. with layers. The system is made from elongated molecules grouped in layers. C stays for tilted, which means that the long axis of the molecules form a nonzero angle with respect to the normal of the layers. Finally, the star * means that the molecules are chiral.

Second, why is $\mathrm{SmC}^{*}$ a liquid crystal? The molecules in the same layer are behaving like a liquid, since there is no positional order and they can freely move in the plane. There is however an orientational order, since all the molecules in the same layer point toward the same direction.

Clearly, there are two angles which describe the direction of each molecule, the tilt angle $\theta$ (the angle between the direction of the molecule and the normal to its layer) and the azimuthal angle $\phi$ (the angle between the projection of the molecule's direction on the layer's plane and a specified fixed direction in the same plane). As we said before, the tilt angle $\theta$ is fixed for all the molecules in the whole liquid crystal. As regarding the azimuthal angle $\phi$, it is equal for all the molecules in the same layer, but it differs from layer to layer. However, the difference of this angle between any two neighbor layers is fixed in the whole system, i.e. the difference $\alpha=\phi_{j+1}-\phi_{j}$ for layers $j+1$ and $j$ does not depend on the choice of $j$. So, the whole $\mathrm{SmC}^{*}$ liquid crystal can be described by two angles, $\theta$ and $\alpha$, which are the order parameters of the system.

The system is in a crystal phase below $T \approx 110^{\circ} \mathrm{C}$, while above $T \approx 120^{\circ}$ $\mathrm{C}$ the tilt angle $\theta \approx 0$, so that $\alpha$ is not defined. The interesting regime is thus between these two temperatures, where the polar angle is constant, $\theta \approx 20^{\circ}$.

What is important is the behavior of the azimuthal angle as function of the temperature, $\alpha(T)$. It comes out that $\alpha(T)$ varies in this range, and that at different temperatures it changes abruptly and discontinuously. This signals first order phase transitions. So the system undergoes through different phases. The interesting point is that this change is not monotone: rising the temperature one goes through phases with $\alpha \neq 0$ to a phase with $\alpha=0$ and later to a phase with $\alpha \neq 0$ again. This is clearly similar to the case of the Rochelle salt and the same conclusions can be applied also here.

\section{$3 \quad$ Field Theory}

The known examples of symmetry nonrestoration in field theory can be divided into three different classes, which will be described below.

The prototype case. This case was first studied by Weinberg $\mathrm{O}$ and later on by Mohapatra and Senjanovic 6 , who were the first to recognize the 
important phenomenological applications of the phenomenon of symmetry nonrestoration at high temperature.

The simplest model consists of two real scalar fields and a $Z_{2} \times Z_{2}$ symmetry, with the zero temperature potential given by

$$
V=\frac{\lambda_{1}}{4} \phi_{1}^{4}+\frac{\lambda_{2}}{4} \phi_{2}^{4}+\frac{\lambda}{2} \phi_{1}^{2} \phi_{2}^{2}+\frac{\mu_{1}^{2}}{2} \phi_{1}^{2}+\frac{\mu_{2}^{2}}{2} \phi_{2}^{2} .
$$

The boundedness from below of this potential requires that $\lambda_{1,2}>0$ and $\lambda_{1} \lambda_{2}>\lambda^{2}$. At high temperature $\left(T>>\mu_{1,2}\right)$ one uses the general one-loop formula to calculate the leading correction:

$$
\Delta V_{T}=\frac{T^{2}}{24} \sum_{i} \frac{\partial^{2} V}{\partial \phi_{i}^{2}}=\frac{T^{2}}{24}\left(3 \lambda_{1}+\lambda\right) \phi_{1}^{2}+\frac{T^{2}}{24}\left(3 \lambda_{2}+\lambda\right) \phi_{2}^{2} .
$$

One can now choose the parameters so that $\left(3 \lambda_{1}+\lambda\right)<0$ and obtain a nonzero vev for the first field, $\left\langle\phi_{1}\right\rangle \neq 0$, spontaneously breaking in this way the first discrete symmetry $Z_{2}$. Due to the boundedness conditions the same can not be done for the second $Z_{2}$. In fact $\left(3 \lambda_{2}+\lambda\right)$ must now be positive, so the second field does not develop a nonzero vev, i.e. $\left\langle\phi_{2}\right\rangle=0$. The reason for the idea to work is the choice of a large negative Higgs coupling $(\lambda)$.

Since at a very high temperature, the temperature itself is the only mass scale in the problem, the nonzero vev of $\phi_{1}$ must be proportional to the temperature. So, we have an example of symmetry nonrestoration, which persists at arbitrary high temperatures.

What happens with higher order terms or nonperturbative contributions? In the case of global symmetries different techniques have shown that symmetry nonrestoration is a possible phenomenon (see however some opposite claimst), but that the parameter space where this can happen tends to be smaller than at one loop 6 . In the case of gauge symmetries many calculations indicates the opposite, i.e. that for physical walnes of the gauge couplings symmetry restoration is probably unavoidable 610.11.

Flat directions. This case most naturally happens in supersymmetry, which is particularly welcome, since the trick of the previous section cannot be applied to susy models. In fact there is a no-go theorem 12, which states that at high enough temperature any internal symmetry gets always restored in renormalizable susy models. This is because the coupling constants are much more constrained in susy than in ordinary models, so that the small island of parameter space which allows symmetry nonrestoration in ordinary theories completely disappears when one looks at the supersymmetric subspace. The same seems to be true also in nonrenormalizable susy models 13 . 
To avoid the no-go theorem one can consider a field which is not in thermal equilibrium with the rest of the system 14 , i.e. its interaction is negligible compared to the expansion rate of the universe. This means essentially that its coupling must be suppressed by inverse powers of a large cutoff. For this reason it does not get necessarily a positive high temperature mass term, which is the main reason for symmetry restoration.

In ordinary nonsupersymmetric models nothing forbids a term $|\phi|^{4}$, which would again put the field $\phi$ in equilibrium with the system. Here is where supersymmetry plays its role. It can not only easily forbid dangerously strong terms, but it is even very natural to have plenty of flat or quasi-flat directions, which are not coupled or extremely weakly coupled to the rest of the system.

It has to be stressed that one needs supersymmetry to naturally have flat directions and high temperature to lift them and stabilize their vevs at large nonzero values. The mechanism is very simple and natural because of the existence of a very large number of flat directions in the minimal supersymmetric standard model (MSSM) and other phenomenologically interesting models.

Large external charge. If one puts large enough charge in a system 15.16, thermal excitations cannot 'absorb' all of it and it must be 'stored' in the vacuum, which thus becomes nontrivial. This is another easy way to give a nonzero vev to a scalar field. It is universal, being valid in both supersymmetric or ordinary models with gauge and/or local symmetries.

What happens is that a scalar particle gets a negative mass term $-\mu^{2}|\phi|^{2}$ with $\mu$ the field's chemical potential. This term tends to give a nonzero vev to $\phi$, an opposite behavior with respect to the pure temperature contribution to the mass term, $+T^{2}|\phi|^{2}$. For a chemical potential (or, better, charge density) bigger than a critical one, the total mass term for $\phi$ becomes negative and $\phi$ acquires a nonzero vev, thus breaking some symmetry, since any field with a chemical potential must transform nontrivially under some group.

However it is not a priori necessary that $\phi$ transforms nontrivially under exactly that symmetry, which originates the nonzero charge density. This is welcome, since one can achieve in this way a nonzero Higgs vev in the standard model at very high temperature with a large lepton number density in the universe although the Higgs boson does not carry a lepton charge 15.12$] 18$ ! And this is exactly what could have happened in the early universe. The critical charge needed for the weak $\mathrm{SU}(2)_{L}$ to be broken at any high enough temperature comes out to be of order $n_{L} \approx T^{3}$, i.e. of the order of the entropy density. Since the standard model has only one Higgs doublet, one cannot break also the electromagnetic $\mathrm{U}(1)$. This can be however easily achieved in the MSSM due to the presence of many scalars 19 .

There are still three issues we want to explain. First, such large lepton 
charges in the universe are allowed by the experimental data 20 . Second, even if strictly speaking the lepton number itself is not conserved in the standard model, due to the breaking of weak $\mathrm{SU}(2)_{L}$ sphalerons are not operative and the universe behaves similarly as it does at (almost) zero temperature today, i.e. effectively conserves lepton (and baryon) number 17. Third, the problem of producing such a large lepton number still remains. There has been some attempts 21 in this direction using the Affleck-Dine mechanism, as well as possible explanations of the small baryon number 22 .

\section{Cosmological applications}

The above ideas can be used in various mechanisms of baryogenesis 23 and inflation 24 as well as to solve the following cosmological problems:

The monopole problem. During a phase transition from a symmetry group G (take SU(5) for example) to a lower one $\mathrm{H}$ (the standard model $\left.\mathrm{SU}(3)_{C} \times \mathrm{SU}(2)_{L} \times \mathrm{U}(1)_{Y}\right)$, monopoles are created via the Kibble mechanism in many grand unified extensions of the standard model. Since monopoles created at the breaking scale of the grand unified theory would survive till today with at least ten orders of magnitude more energy density than baryons 25 , such a possibility is clearly unacceptable, and is referred to as the monopole problem. An elegant solution to this problem is to spontaneguslypreak the initial group

$\mathrm{G}$ or at least the $\mathrm{U}(1)$ factor in the final group H6,26, 27, 10. This solution does not depend on the specific inflationary model used, and it does not pose any constraint on it, so that now the reheating temperature can be also large.

The domain wall problem. This problem 28 is very similar in nature to the monopole one. The only difference is in the groups involved, so that it appears only when the vacuum manifold is disconnected. A typical example is a model with a discrete group $Z_{2}$. Again, too energetically domain walls get created during a phase transition between a phase with restored $Z_{2}$ (high $T$ ) and a phase with spontaneously broken $Z_{2}$ (low $T$ ). As before, if $Z_{2}$ is instead spontaneously broken at any temperature, there is no phase transition and thus no domain wall problem 6,29 . Such a solution is welcome whenever we have theories with spontaneously broken $P, C P$ or Peccei-Quinn symmetries.

The false vacuum problem. Take for example the case of supersymmetric SU(5). The effective potential has at low temperature three degenerate vacua, the $\mathrm{SU}(5)$ conserving, the one with the symmetry $\mathrm{SU}(4) \times \mathrm{U}(1)$ and the standard model vacuum $\mathrm{SU}(3) \times \mathrm{SU}(2) \times \mathrm{U}(1)$, depending on the vev of the adjoint Higgs in the representation 24. If one assumes $\mathrm{SU}(5)$ symmetry restoration at high temperature, the universe would remain in this same vacuum through all the history of the universe, since the barrier between different 
vacua are too high to tunnel through. If this were true, we could never live in our universe with the standard model symmetry group 30 . Needless to say, a possible and elegant solution is given by symmetry nonrestoration: if $\mathrm{SU}(5)$ has been spontaneously broken at any high temperature to our standard model gauge group, no tunneling is necessary and the problem does not appear.

\section{Acknowledgments}

It is a pleasure to thank the organizers of COSMO99 as well as Mojca Čepič, Gia Dvali, Alejandra Melfo, Toni Riotto and especially Goran Senjanović. This work was supported by the Ministry of Science and Technology of the Republic of Slovenia and by the Packard Foundation 99-1462 fellowship.

\section{References}

1. G. Senjanović, COSMO 97, ed. L. Roszkowski (World Scientific, Singapore, 1998), 437, hep-ph/9805361; B. Bajc, COSMO 98, ed. D. Caldwell (AIP, New York, 1999), 220, hep-ph/9902470.

2. F. Jona and G. Shirane, Ferroelectric Crystals (Pergamon, Oxford, 1962), 280.

3. S. Weinberg, Phys. Rev. D 9, 3357 (1974).

4. M. Čepič and B. Žekš, Mol. Cryst. Liq. Cryst. 263, 61 (1995).

5. P. Mach et al, Phys. Rev. Lett. 81, 1015 (1998).

6. R.N. Mohapatra and G. Senjanović, Phys. Rev. Lett. 42, 1651 (1979); Phys. Rev. D 20, 3390 (1979).

7. Y. Fujimoto and S. Sakakibara, Phys. Lett. B 151, 260 (1985); G. Bimonte, D. Iniguez, A. Tarancon and C.L. Ullod, Phys. Rev. Lett. 81, 750 (1998), hep-lat/9802022.

8. G. Bimonte and G. Lozano, Phys. Lett. B 366, 248 (1996), hepth/9507079; J. Orloff, Phys. Lett. B 403, 309 (1997), hep-ph/9611398; T.G. Roos, Phys. Rev. D 54, 2944 (1996), hep-th/9511073; M. Pietroni, N. Rius and N. Tetradis, Phys. Lett. B 397, 119 (1997), hep-ph/9612205; G. Amelino-Camelia, Phys. Lett. B 388, 776 (1996), hep-ph/9610262, M.B. Pinto and R.O. Ramos, hep-ph/9912273.

9. K. Jansen and M. Laine, Phys. Lett. B 435, 166 (1998), hep-lat/9805024; G. Bimonte, D. Iniguez, A. Tarancon and C.L. Ullod, Nucl. Phys. B 559, 103 (1999), hep-lat/9903027.

10. G. Dvali, A. Melfo and G. Senjanović, Phys. Rev. Lett. 75, 4559 (1995), hep-ph/9507230.

11. G. Bimonte and G. Lozano, Nucl. Phys. B 460, 155 (1996), hepth/9509060; M.B. Gavela, O. Péne, N. Rius and S. Vargas-Castrillon, 
Phys. Rev. D 59, 025008 (1999), hep-ph/9801244.

12. H. Haber, Phys. Rev. D 46, 1317 (1982); M. Mangano, Phys. Lett. B 147, 307 (1984).

13. B. Bajc, A. Melfo and G. Senjanović, Phys. Lett. B 387, 796 (1996), hep-ph/9607242; G. Dvali and K. Tamvakis, Phys. Lett. B 378, 141 (1996), hep-ph/9602336.

14. G. Dvali and L. Krauss, preprint CWRU-P31-98, hep-ph/9811298; B. Bajc and G. Senjanović, preprint IJS-TP-98-24, hep-ph/9811321.

15. A.D. Linde, Phys. Rev. D 14, 3345 (1976).

16. H.E. Haber and H.A. Weldon, Phys. Rev. D 25, 502 (1982); K.M. Benson, J. Bernstein and S. Dodelson, Phys. Rev. D 44, 2480 (1991); A. Riotto and G. Senjanović, Phys. Rev. Lett. 79, 349 (1997), hepph/9702319; A. Riotto, Nucl. Phys. Proc. Suppl. 62, 253 (1998), hep-ph/9706296.

17. J. Liu and G. Segre, Phys. Lett. B 338, 259 (1994).

18. B. Bajc, A. Riotto and G. Senjanović, Phys. Rev. Lett. 81, 1355 (1998), hep-ph/9710415.

19. B. Bajc and G. Senjanović, hep-ph/9907552.

20. H.-S. Kang and G. Steigman, Nucl. Phys. B 372, 494 (1992); W.H. Kinney and A. Riotto, Phys. Rev. Lett. 83, 3366 (1999), hep-ph/9903459; J. Lesgourgues and S. Pastor, Phys. Rev. D 60, 103521 (1999), hep$\mathrm{ph} / 9904411$.

21. A. Casas, W.-Y. Cheng and G. Gelmini, Nucl. Phys. B 538, 297 (1999), hep-ph/9709289.

22. J. McDonald, Phys. Lett. B 463, 225 (1999), hep-ph/9907358; hep$\mathrm{ph} / 9908300$.

23. R.N. Mohapatra and G. Senjanović, Phys. Rev. D 21, 3470 (1980); V.A. Kuzmin, M.E. Shaposhnikov and I.I. Tkachev, Phys. Lett. B 105, 159 and 167 (1981), Nucl. Phys. B 196, 29 (1982); S. Dodelson and L.M. Widrow, Phys. Rev. Lett. 64, 340 (1990); S. Dodelson, B.R. Greene and L.M. Widrow, Nucl. Phys. B 372, 467 (1992).

24. J.-w. Lee and I.-g. Koh, Phys. Rev. D 54, 7153 (1996), hep-ph/9506415.

25. J. Preskill, Phys. Rev. Lett. 43, 1365 (1979).

26. P. Langacker and S.-Y. Pi, Phys. Rev. Lett. 45, 1 (1980).

27. P. Salomonson, B.S. Skagertan and A. Stern, Phys. Lett. B 151, 243 (1985).

28. Ya.B. Zeldovich, I.Yu. Kobzarev and L.B. Okun, JETP 40, 1 (1974).

29. G. Dvali and G. Senjanović, Phys. Rev. Lett. 74, 5178 (1995), hep$\mathrm{ph} / 9501387$.

30. S. Weinberg, Phys. Rev. Lett. 48, 1776 (1982). 